\title{
Kepler-1649b: An Exo-Venus in the Solar Neighborhood
}

\author{
Isabel Angelo ${ }^{1,2,3}$, Jason F. Rowe ${ }^{1,2,4,5}$, Steve B. Howell ${ }^{2}$, Elisa V. Quintana ${ }^{2}$, Martin Still ${ }^{6}$, Andrew W. Mann ${ }^{7}$, \\ Ben Burningham ${ }^{2,8}$, Thomas Barclay ${ }^{2,6}$, David R. Ciardi ${ }^{9}$, Daniel Huber ${ }^{1,10,11}$, and Stephen R. Kane ${ }^{12}$ \\ ${ }^{1}$ SETI Institute, Mountain View, CA 94043, USA; isabelangelo@berkeley.edu \\ ${ }^{2}$ NASA Ames Research Center, Moffett Field, CA 94035, USA \\ ${ }^{3}$ Department of Astronomy, University of California, Berkeley, CA, 94720, USA \\ ${ }^{4}$ Institut de recherche sur les exoplanètes, iREx, Département de physique, Université de Montréal, Montréal, QC, H3C 3J7, Canada \\ ${ }^{5}$ Department of Physics, Bishops University, 2600 College Street, Sherbrooke, QC, J1M 1Z7, Canada \\ ${ }^{6}$ Bay Area Environmental Research Institute, 625 2nd Street, Suite 209, Petaluma, CA 94952, USA \\ ${ }^{7}$ Department of Astronomy, The University of Texas at Austin, Austin, TX 78712, USA \\ ${ }^{8}$ Centre for Astrophysics Research, School of Physics, Astronomy and Mathematics, University of Hertfordshire, Hatfield AL10 9AB, UK \\ ${ }^{9}$ NASA Exoplanet Science Institute/Caltech, Pasadena, CA, USA \\ ${ }^{10}$ Sydney Institute for Astronomy (SIfA), School of Physics, University of Sydney, NSW 2006, Australia \\ ${ }^{11}$ Stellar Astrophysics Centre, Department of Physics and Astronomy, Aarhus University, Ny Munkegade 120, DK-8000 Aarhus C, Denmark \\ ${ }^{12}$ Department of Physics \& Astronomy, San Francisco State University, 1600 Holloway Avenue, San Francisco, CA 94132, USA \\ Received 2016 October 25; revised 2017 February 13; accepted 2017 February 15; published 2017 March 17
}

\begin{abstract}
The Kepler mission has revealed that Earth-sized planets are common, and dozens have been discovered to orbit in or near their host star's habitable zone. A major focus in astronomy is to determine which of these exoplanets are likely to have Earth-like properties that are amenable to follow-up with both ground- and future space-based surveys, with an ultimate goal of probing their atmospheres to look for signs of life. Venus-like atmospheres will be of particular interest in these surveys. While Earth and Venus evolved to have similar sizes and densities, it remains unclear what factors led to the dramatic divergence of their atmospheres. Studying analogs to both Earth and Venus can thus shed light on the limits of habitability and the potential for life on known exoplanets. Here, we present the discovery and confirmation of Kepler-1649b, an Earth-sized planet orbiting a nearby M5V star that receives incident flux at a level similar to that of Venus. We present our methods for characterizing the star, using a combination of point-spread function photometry, ground-based spectroscopy, and imaging, to confirm the planetary nature of Kepler-1649b. Planets like Kepler-1649b will be prime candidates for atmospheric and habitability studies in the next generation of space missions.
\end{abstract}

Key words: planets and satellites: terrestrial planets

\section{Introduction}

The Kepler mission was designed to measure the frequency and sizes of extrasolar planets (Borucki et al. 2010), with a primary goal of detecting other Earth-sized planets that could potentially be habitable. In our solar system, both Earth and Venus evolved to have comparable sizes and bulk densities, yet the evolution of their atmospheres diverged dramatically such that only Earth developed conditions conducive to the emergence of life. It remains unclear which aspects of Earth's development were key in acquiring and maintaining a hospitable atmosphere. Finding and characterizing both Earth and Venus analogs around other stars could shed light on these differences.

Kepler has been successful in finding Earth-size planets in the habitable zones of their host stars (Quintana et al. 2014; Torres et al. 2015). A super-Earth in a Venus-like orbit and dozens of small planet candidates that could potentially have Venus-like atmospheres have also been discovered (Barclay et al. 2013a; Kane et al. 2013, 2013). In this paper we confirm the planetary nature of Kepler-1649b (KOI-3138.01), an Earthsized planet that receives flux from its host star that is comparable to that received by Venus.

Kepler-1649 appears in the Kepler Input Catalog (KIC; Brown et al. 2011) as KIC 6444896 with a brightness of $17.131 \mathrm{mag}$ in the Kepler bandpass $(K p)$ and has a relatively high proper motion of 0 "! $157 \mathrm{yr}^{-1}$ (Lépine \& Shara 2005). It was not selected as a prime mission target (Batalha et al. 2010) but was proposed as part of Cycle 2 of the NASA Guest Observer (GO) Program (GO20031) to search for gravitational lensing in the Kepler field of view (di Stefano 2012). Through the GO program, long-cadence (30-minute) observations covering quarters Q6-Q9 were collected for 1 yr. A transit with a period of 8.7 days was detected, and the target was given the designation Kepler Object of Interest (KOI) 3138 (Burke et al. 2014). After the discovery and dispositioning of the transit event, the target was added to the prime exoplanet target list for Q12-Q17.

In the Q1-Q12 catalog (Rowe et al. 2015), Kepler-1649b was noted as an interesting cool sub-Earth-radius planet candidate in an 8.7-day period around a cool $\mathrm{M}$ dwarf $\left(T_{\text {eff }}=2703\right.$ ) based on broadband photometric colors (Huber et al. 2014). The fitted value of the mean stellar density $\left(\rho_{\star}\right)$ of $70 \pm_{42}^{25} \mathrm{~g} \mathrm{~cm}^{-3}$ and short transit duration, $1.04 \pm 0.10 \mathrm{hr}$ as reported in the Q1-Q12 catalog, were consistent with the cool dwarf characterization of the host star.

While Kepler-1649b shares a similar size and incident flux as Venus, it orbits a nearby (219 lt-yr) M5V star that is about one-quarter of the size and mass of our Sun. Estimates on the size of this planet evolved as better constraints on the star's properties were attained over a period of several years from its initial detection. Although Kepler photometry provides the orbital period and the planet's size relative to its host star to high precision, characterizing the transiting exoplanet is 
typically more limited since its fundamental properties depend critically on properties of the host star. For the bulk of the Kepler planet sample, these stellar properties are based on matching broadband photometric measurements to stellar evolution models with various choices for priors that may or may not account for observational biases (Brown et al. 2011; Batalha et al. 2013; Huber et al. 2014). Improving these stellar parameters with better diagnostics such as spectroscopy can help us learn about systematics that may skew our interpretation of the Kepler sample. Additionally, follow-up highresolution imaging of Kepler planet candidates is crucial for constraining properties of the planet system and its stellar host (s), particularly because $50 \%$ of planet hosts are likely to be binary (Horch et al. 2014).

The planet candidate in the Kepler-1649 (KOI-3138) system was first listed in the Kepler Q1-Q8 catalog (Burke et al. 2014) with a stellar radius of $0.6 R_{\odot}$ and a planet size of $4.6 R_{\oplus}$. Soon after the Q1-Q8 KOI release, there was a substantial effort from the Kepler Stars Working Group to improve global estimates of the stellar parameters. Most stars in the Kepler sample, including Kepler-1649, were refit using Dartmouth models (Dotter et al. 2008). This gave a smaller estimated stellar radius of $0.12 R_{\odot}$ (Huber et al. 2014). Matching the new stellar parameters to improved transit models gave a planet with a radius of $R_{\mathrm{p}}=0.57 R_{\oplus}$ receiving an incident flux $(S)$ of 0.47 relative to Earth around a host star with $T_{\text {eff }}=2703 \mathrm{~K}$, as noted in the Q1-Q12 catalog (Rowe et al. 2015).

With the revised stellar parameters, the Kepler-1649 system appeared to host a Mars-sized planet around a cool, nearby $M$ dwarf with incident bolometric flux levels similar to Mars. These properties would make this planet the first "exoMars" and would add to the small sample of potentially rocky planets transiting in or near the habitable zone of nearby M dwarfs. We obtained follow-up spectroscopy and imaging to verify the planetary nature of the transiting planet and better constrain stellar properties. Such imaging could also potentially detect companions or background stars that indicate a planet radius larger than that determined by the transit depth. We found that the host star is significantly larger and hotter than previously estimated. The radius and incident flux levels of the planet increased to $1.08 R_{\oplus}$ and $2.30 S_{\oplus}$, respectively. Revised stellar properties that necessitate recharacterization of planet properties are not unique to the Kepler-1649 system, but rather a common occurrence for Kepler systems (Everett et al. 2013; Huber et al. 2013; Gaidos et al. 2016). This case provides an example of the caution needed when constraining a planet's size based on various star catalogs, as well as the value of follow-up observations to improve estimates of the host star properties.

Herein we present our confirmation of Kepler-1649b using transit and stellar models combined with ground-based observations. In Section 2 we present our ground-based spectroscopic follow-up observations and classification of Kepler-1649b. In Section 3 we present our technique of point-spread function (PSF) extracted photometry and lightcurve modeling to constrain the planet properties of Kepler1649b. Our validation of Kepler-1649b as a planet using ground-based follow-up imaging is presented in Section 4. Finally, we summarize our results and comment on relative comparisons to Venus in Section 5.
Table 1

System Parameters for Kepler-1649

\begin{tabular}{lll}
\hline \hline Parameter & Value & Notes \\
\hline Transit and orbital parameters: & & \\
Orbital period $P$ (days) & $8.689090 \pm 0.000024$ & $\mathrm{~A}$ \\
Midtransit time $E$ (HJD) & $2454966.2348 \pm 0.0026$ & $\mathrm{~A}$ \\
Scaled semimajor axis $a / R_{\star}$ & $60.6 \pm 8.1$ & $\mathrm{~A}$ \\
Scaled planet radius $R_{\mathrm{p}} / R_{\star}$ & $0.0391_{-0.0022}^{+0.0014}$ & $\mathrm{~A}$ \\
Impact parameter $b \equiv a$ cos $i / R_{\star}$ & $0.34_{-0.34}^{+0.15}$ & $\mathrm{~A}$ \\
Orbital inclination $i$ (deg) & $89.57 \pm 0.32$ & $\mathrm{~A}$ \\
Derived stellar parameters: & & \\
Effective temperature $T_{\mathrm{eff}}(\mathrm{K})$ & $3240 \pm 61$ & $\mathrm{~B}$ \\
Spectroscopic gravity $\log g(\mathrm{cgs})$ & $4.98 \pm 0.22$ & $\mathrm{~B}$ \\
Metallicity [Fe/H] & $-0.15 \pm 0.11$ & $\mathrm{~B}$ \\
Mass $M_{\star}\left(M_{\odot}\right)$ & $0.219 \pm 0.022$ & $\mathrm{C}$ \\
Radius $R_{\star}\left(R_{\odot}\right)$ & $0.252 \pm 0.039$ & $\mathrm{C}$ \\
Planetary parameters: & & \\
Radius $R_{\mathrm{p}}\left(R_{\oplus}\right.$, equatorial) & $1.08 \pm 0.15$ & $\mathrm{~A}, \mathrm{~B}, \mathrm{C}$ \\
Orbital semimajor axis $a(\mathrm{au})$ & $0.0514 \pm 0.0028$ & $\mathrm{D}$ \\
Incident flux $\left(S_{\oplus}\right)$ & $2.30 \pm 0.65$ & $\mathrm{D}$ \\
\hline
\end{tabular}

Note. A: based on Kepler photometry. B: based on an analysis of the Palomar spectra. C: based on stellar evolution tracks. D. based on Newton's version of Kepler's third law and total mass.

\section{Spectroscopic Observations and Stellar Classification}

Spectroscopic observations of Kepler-1649 were made on 2015 February 11 with the Double-Beam spectrograph attached to the 200" Hale reflector at the Mount Palomar Observatory. The dichroic filter D-68 was used to split light between the blue and red arms near $7000 \AA$. The blue arm used a 1200 line $\mathrm{mm}^{-1}$ grating providing $R \sim 7700$ and covered $\sim 2500 \AA$ of the spectrum, 4200-7000 $\AA$. The red arm also used a 1200 line $\mathrm{mm}^{-1}$ grating providing $R \sim 9000$ and covered $\sim 2500 \AA$ of the spectrum, 7000-9500 $\AA$. The spectra have a dispersion of approximately $0.82 \AA$ pixel $^{-1}$ across the bandpass. The slit width was set to $1^{\prime \prime}$, the integration time was $300 \mathrm{~s}$, and the usual procedures of observing spectrophotometric stars and arc lamps were adhered to. Red spectra were wavelength-calibrated with an HeNeAr lamp, while the blue arm used a FeAr lamp. The nights were clear and provided stable seeing near $1^{\prime \prime}$. Data reduction was done using IRAF two- and one-dimensional routines for spectroscopic data and produced a final onedimensional spectrum for each observation, as shown in Figure 1. From analysis of these spectra, we were able to derive the effective temperature $\left(T_{\text {eff }}\right)$, stellar radius $\left(R_{\star}\right)$, stellar metallicity $([\mathrm{Fe} / \mathrm{H}])$, and stellar gravity $(\log g)$, as described in the following paragraphs. Our results are shown in Table 1.

We determined $T_{\text {eff }}$ for Kepler-1649 following the method of Mann et al. (2013b), which we briefly summarize here. We compared our optical spectrum to a grid of PHOENIX BTSETTL models ${ }^{13}$ (Allard et al. 2011). We masked out regions of the spectrum that are poorly reproduced by atmospheric models. The fit included six nuisance parameters to account for errors in wavelength and flux calibration and the offset between the blue and red arms of the spectrum. We derived an error on $T_{\text {eff }}$ based on the scatter in the model fits and a comparison between $T_{\text {eff }}$ values derived this way and those determined

\footnotetext{
${ }^{13}$ https://phoenix.ens-lyon.fr/Grids/BT-Settl/CIFIST2011
} 

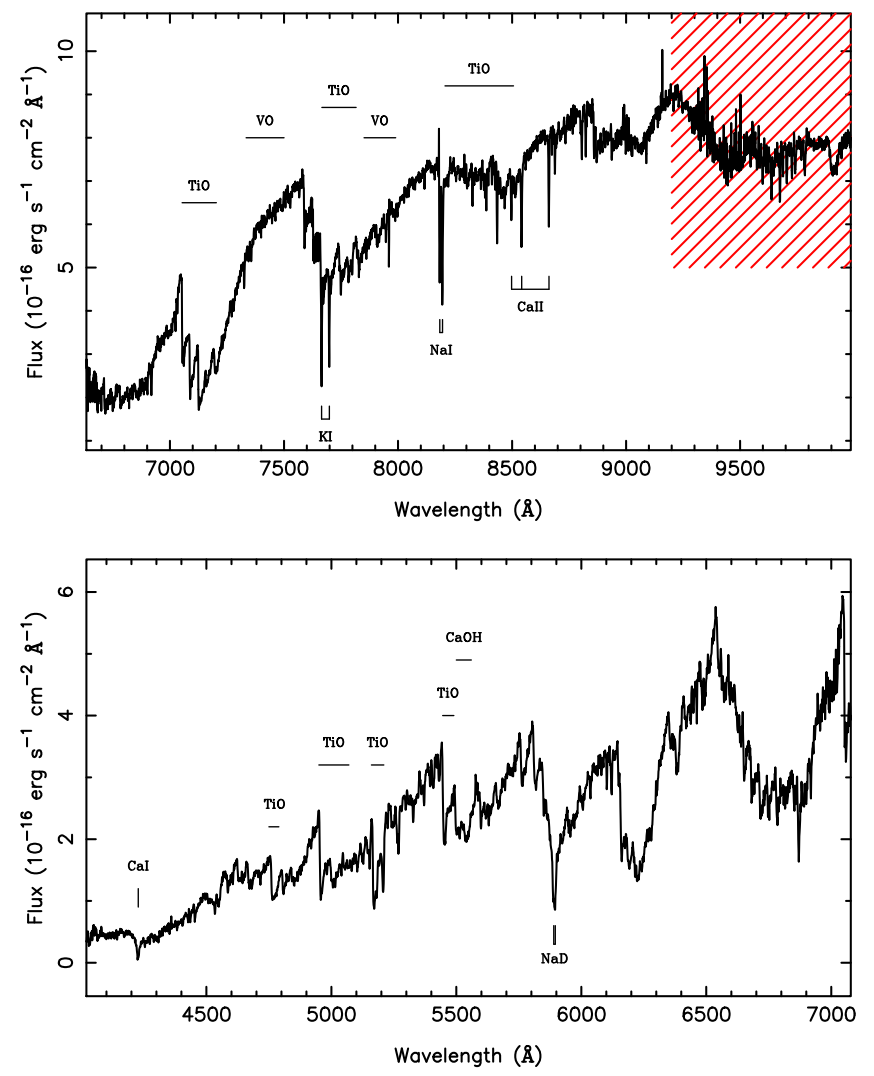

Figure 1. Spectrum of Kepler-1649 collected using the Double-Beam spectrograph on the $200^{\prime \prime}$ Hale telescope at Palomar Observatory. The bottom panel shows the blue beam and the top panel the red beam. The spectrum is consistent with a classification of a mid-M dwarf star. The red hatched region is compromised because it is dominated by telluric lines and was not used in our analysis.

empirically from long-baseline optical interferometry (Boyajian et al. 2012). Our final $T_{\text {eff }}$ is $3240 \pm 61 \mathrm{~K}$.

We combine our optical spectrum with the formula from Mann et al. (2013a) to determine the host star's [Fe/H]. Mann et al. (2013a) present empirical relations between the strength of atomic lines in visible and near-infrared $\mathrm{M}$ dwarf spectra and the metallicity of the host star, calibrated using a set of wide $\mathrm{FGK}+\mathrm{M}$ dwarf binaries. Using the calibration for visiblewavelength lines, we calculated $[\mathrm{Fe} / \mathrm{H}]=-0.15 \pm 0.11$.

We calculated the stellar radius $\left(R_{\star}\right)$ from our $T_{\text {eff }}$ and $[\mathrm{Fe} / \mathrm{H}]$ using the relations from Mann et al. (2015), which are based on nearby single stars with precise $(<5 \%)$ parallaxes. Our stellar radius errors account for errors in both $T_{\text {eff }}$ and $[\mathrm{Fe} / \mathrm{H}]$ and the scatter in relations from Mann et al. (2015). We used these relations in conjunction with stellar evolution tracks to calculate a stellar radius of $R_{\star}=0.252 \pm 0.039 R_{\odot}$. We also used isochrone model fits to determine the stellar gravity $\log g=4.98 \pm 0.22$ as outlined in Teske et al. (2015) to arrive at a mass $M_{\star}=0.219 \pm 0.022 M_{\odot}$.

\section{Planet Properties}

Photometry provided by the Kepler project is based on apertures meant to maximize the signal-to-noise ratio based on positions and apparent magnitudes from the KIC. However, it has previously been shown for targets fainter than 15th16th mag in the Kepler bandpass that photometry based on a model of the PSF is more precise (Rappaport et al. 2014). This form of photometry utilizes the Kepler pixel response function
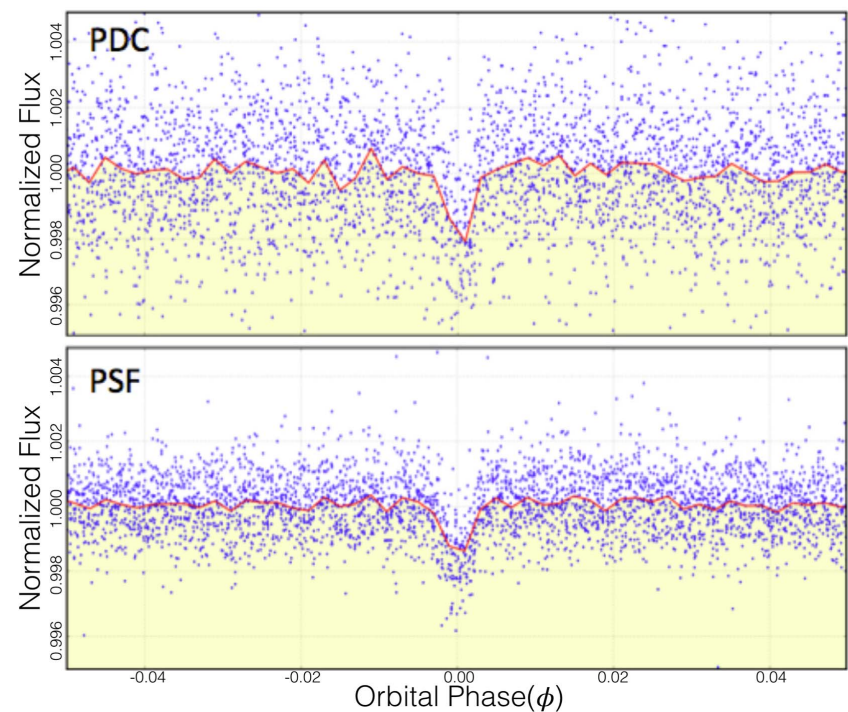

Figure 2. The 10-quarter Kepler light curve collected from Kepler-1649. The light curve has been extracted from calibrated pixels in two separate ways: all pixels within the photometric aperture defined by the Kepler pipeline are summed and then passed through the PDC algorithm (top), and a PSF model is fit to all pixels within the mask (bottom). Signals of astrophysical origin and systematics on timescales $>1$ day have been removed and the light curves normalized. Both time series have been folded on an 8.68904-day orbital period with zero phase corresponding to BJD 2,454,966.2406. Blue dots are individual observations, and the red line is the same data median-averaged into 500 uniformly sized phase bins.

that the mission has archived at the Mikulski Archive for Space Telescopes (MAST; Bryson et al. 2010).

Utilizing the method described in Section 3.1 of Rappaport et al. (2014), we modeled Kepler-1649 and six stars near the target that had initial position and brightness values based on either the KIC or two different multicolor surveys of the Kepler field (Everett et al. 2012; Greiss et al. 2012) using software provided by the Kepler GO Office (Still \& Barclay 2012). The scatter integrated over the transit duration for Kepler-1649b of $1.04 \mathrm{hr}$, also known as the $1.04 \mathrm{hr}$ Combined Differential Photometric Precision (CDPP) (Christiansen et al. 2012) from the Kepler data, was $860 \mathrm{ppm}$, whereas our PSF photometry had a scatter of $347 \mathrm{ppm}$, an increase in signal-to-noise ratio of a factor of 2.5. This increase in photometric quality can be seen in Figure 2, where the top panel is the Kepler pipeline derived photometry, summed and passed through a Presearch Data Conditioning (PDC) algorithm (Smith et al. 2012; Stumpe et al. 2012, 2014), and the bottom panel was created using PSF photometry. Our PSF photometry for Kepler-1649 is utilized for the remainder of this paper for deriving planet properties of Kepler-1649b (see Table 1). The full time series of Kepler1649 is shown in Figure 3.

We fit a model to the observed transit of Kepler-1649b to determine the properties of both the planet and host star. We used the transit model of Rowe et al. (2014), which is described by a Keplerian orbit and transit based on the analytical description of Mandel \& Agol (2002) for quadratic limb darkening. The modeled parameters were the orbital period $(P)$, time of first transit $\left(T_{0}\right)$, ratio of the planet and star radius $\left(R_{\mathrm{p}} / R_{\star}\right)$, impact parameter $(b)$, and mean stellar density $\left(\rho_{\star}\right)$. In addition to the model assumption that the mass of the planet is much less than the mass of the star, a circular orbit was adopted to perform our calculations. The best-fit parameters were found 


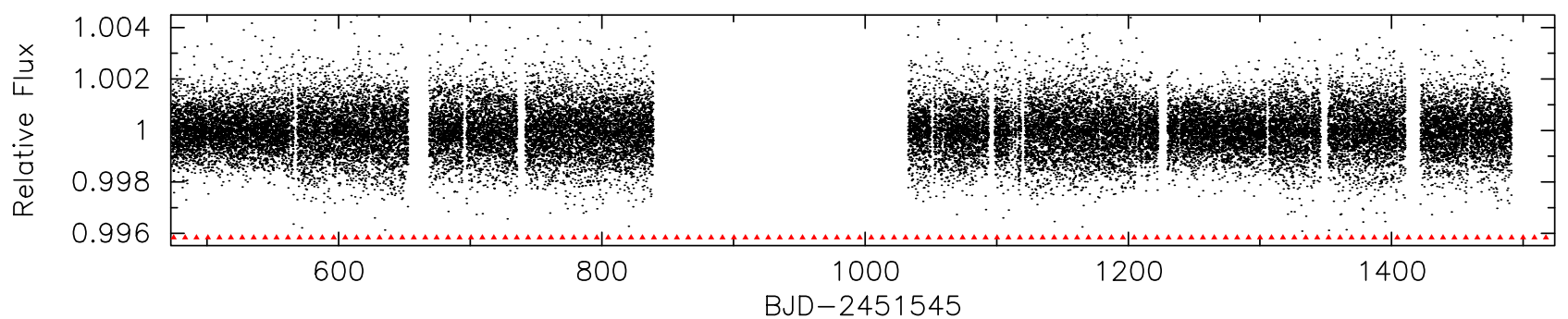

Figure 3. Full Kepler time-series data for Kepler-1649 created using PSF photometry. Transits occur every 8.7 days and are indicated by red triangles. These data cover Kepler observing quarters Q6-Q9 and Q12-Q17. No data were collected during quarters Q10 and Q11, the cause of the gap in the center.

via least-squares analysis. The best-fitting model is shown overplotted on the phase-folded transit data in Figure 4.

To estimate the posterior distribution on each fitted model parameter, we used a Markov chain Monte Carlo (MCMC) approach similar to the procedure outlined in Ford (2005), but modified to better handle correlated variables as implemented in Rowe et al. (2014). The chain generation steps use a combination of Gibbs sampling and vectorized jump via random selection between the two methods. The latter method, vectorized jumps, uses a control set of model parameter sets and scale parameters as described in Gregory (2011). The adopted methods allow for efficient parameter space exploration even with highly correlated variables. The generation of the chains was initially seeded with the best-fitting parameters found from the least-squares fit.

We generated $10^{6}$ Markov chains, the first $20 \%$ of which were discarded as burn-in. The remaining chains were combined into one continuous set and used to calculate the median, standard deviation, and $1 \sigma$ bounds of the distribution centered on the median for each model parameter. The transit and orbital parameters that were derived with the Markov chain include orbital period $(P)$, midtransit time $(E)$, scaled semimajor axis $\left(a / R_{\star}\right)$, scaled planet radius $\left(R_{\mathrm{p}} / R_{\star}\right)$, impact parameter $(b)$, and orbital inclination $(i)$. The orbit was assumed to be circular. We then used the Markov chains to compute model-dependent measurements for the limb-darkened transit depth at midtransit, $\Delta F / F=1783 \pm 101 \mathrm{ppm}$, and full transit duration, $T_{\text {dur }}=1.0357 \pm 0.0966 \mathrm{hr}$.

We convolved our transit model parameters with the stellar parameters to compute the planetary radius $R_{\mathrm{p}}=1.08 \pm 0.15$, orbital semimajor axis $a=0.0514 \pm 0.0028$ au, and flux received by the planet relative to Earth $S=2.30 \pm 0.65$. Our final results are presented in Table 1. Figure 5 shows the range in incident flux and radius for Kepler-1649b from our MCMC analysis, showing that Kepler-1649b has values consistent with the planet Venus, even at the $1 \sigma$ confidence level.

\section{Validation}

We performed a series of analyses and follow-up observations to eliminate the possibility of a false positive and validate Kepler-1649b as a planet. Although false detections due to noise are highly unlikely (Jenkins et al. 2002), it remains possible that our detected transit signal in the Kepler-1649 system is due to some other astrophysical source. Such falsepositive signals may be induced by background or foreground eclipsing binary systems, background or foreground transiting planet systems, or a planet transiting a bound companion to the target star.

Figure 6 shows the $3 \sigma$ regions of stellar magnitude and separation parameter space that we eliminate as potential

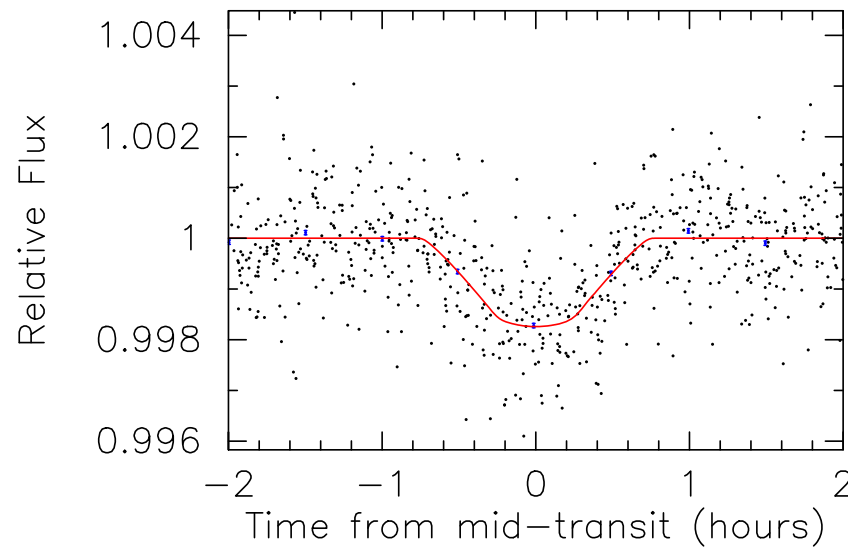

Figure 4. Phase-folded flux time series for Kepler-1649. The flux time series has been folded on the planet's orbital period. Each black point represents one observed datum, and the red curve shows a best-fit transit curve.

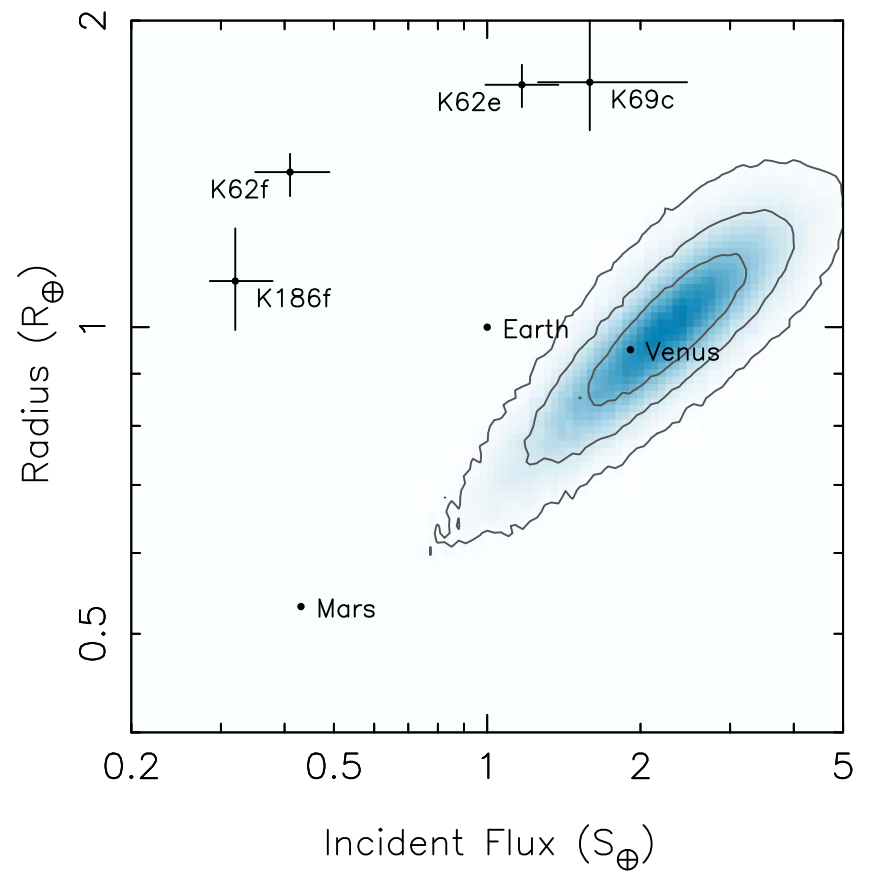

Figure 5. Radius and incident flux for Kepler-1649b based on MCMC analysis. The contours represent $1 \sigma, 2 \sigma$, and $3 \sigma$ confidence levels. Solar system objects Venus, Earth, and Mars are also plotted, as well as a sample with $1 \sigma$ uncertainties of confirmed planets from Kepler: Kepler-186f (Quintana et al. 2014), Kepler-62e (Borucki et al. 2013), Kepler-62f (Borucki et al. 2013), and Kepler-69c (Barclay et al. 2013b).

locations of a false-positive source. The procedures outlined in this section were conducted to assure that no such sources were detected in these regions. We conclude from our observations 


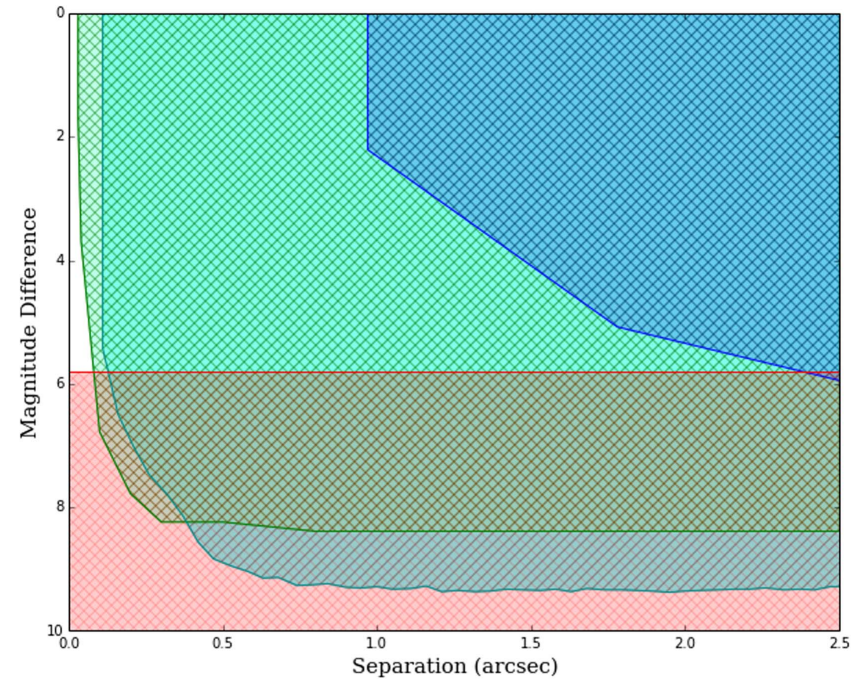

Figure 6. Exclusion zones for Kepler-1649 in which a false-positive source cannot reside. All curves are within $3 \sigma$ certainty. Regions eliminated from Kepler transit data are shown in red, from UKIRT imaging in blue, from speckle data in green, and from AO data in cyan. We cannot rule out the possibility of a false-positive source residing in the white regions of this figure and thus account for it in our false-positive analysis.

that the existence of a false-positive source is highly unlikely, thus allowing our signal to be interpreted as a planet transit associated with the Kepler-1649 system.

To assess the probability of a false positive, we systematically eliminated various regions of parameter space in which a false-positive-inducing source can exist. We began by using the proper motion of the target star to eliminate the chances of a confounding background source. Kepler-1649 is a high-propermotion star $\left(0\right.$.' $\left.157 \mathrm{yr}^{-1}\right)$ and thus resides in a slightly different location in the sky today than it did just decades ago (Lépine \& Shara 2005). We can examine its current location in older images for any possible background stars that may be the source of a false-positive signal. Examination of images from the STScI Digitized Sky Survey taken on 1991 September 06 as part of the POSSII-F Sky Survey ${ }^{14}$ are shown in Figure 7. No background objects are detected at the current location of Kepler-1649 in this image. The POSSII-F image has a plate limit of $R \sim 22.5 \pm 0.4$ (Reid et al. 1991), meaning that a confounding background source would have to be more than $\sim 5$ mag fainter than Kepler-1649. Such a source is likely not bright enough to produce the observed transit, as we show in the next paragraph.

We analyzed the Kepler transit data to place constraints on the magnitude of the transiting object. According to the MCMC transit analysis outlined in Section 3, the detected transit in question has a measured depth of $1783 \pm 101 \mathrm{ppm}$. In the case that the transit was induced by an eclipsing binary system, we can calculate the maximum possible magnitude of the transiting object by assuming that the system undergoes total eclipses (Chaplin et al. 2013). Under this inference, such a depth requires that the source be at most $5.8 \mathrm{mag}$ fainter than the target star in order to fit our transit model. We can thus rule out all nearby stars that are more than 5.8 mag fainter than Kepler1649 as possible transit sources. The red exclusion zone in Figure 6 indicates the region of parameter space in which these stars would reside. Any star not seen with POSSII-F would

\footnotetext{
14 http://stdatu.stsci.edu/cgi-bin/dss_form
}

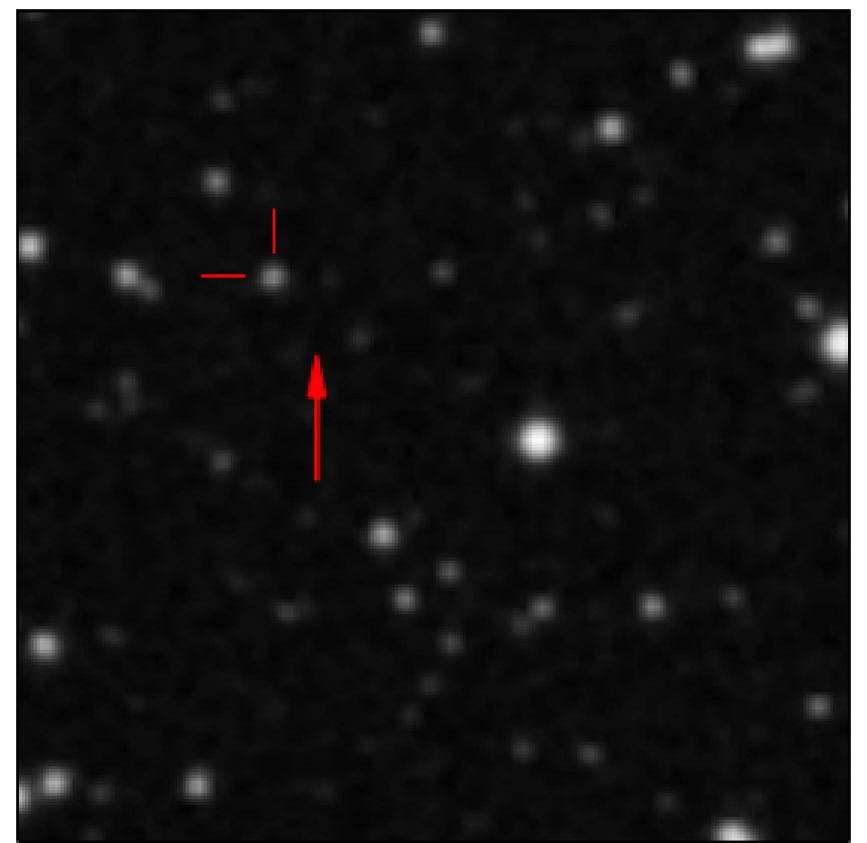

Figure 7. Palomar Observatory Sky Survey (POSSII) image of the region near Kepler-1649 illustrating the lack of background sources. The image is $2.25 \times 2.25$ arcmin on a side, with north up and east to the left. The red arrow indicates the location in which Kepler-1649 currently resides, and the two lines indicate the location of Kepler-1649 when the image was taken. No background objects are detected to a depth of $\sim 22.5$ mag on or near the star's current location.

exist in this region, thus allowing us to completely eliminate the possibility of a background transit source.

We were then left with ruling out the possibility that Kepler$1649 \mathrm{~b}$ orbits a bound companion to Kepler-1649 that cannot be detected in a single spectrum (Teske et al. 2015) or resolved by Kepler or POSSII images, in which case our transiting planet would be larger than the size derived in Section 3. Our next step thus involved inspection of seeing-limited follow-up images of Kepler-1649 to reveal any unresolved companions that could host a transiting planet. The first of these images were taken in the $J$ band by the UK Infrared Telescope (UKIRT). The UKIRT images reveal several stars that fall within a few arcseconds of our target star. All of the resolved stars, given their distance to Kepler-1649, were ruled out as possible transit sources because they did not induce a correlated shift in the photo-center of Kepler-1649 over time, a common characteristic of eclipsing binaries (Bryson et al. 2013). Any false-positive sources farther from our target star would have been seen in our UKIRT images and can thus be ruled out, as indicated by the blue region in Figure 6. There is, however, a seeing limit to images taken with UKIRT of about 0.9 arcsec. We cannot eliminate areas within this seeing limit and therefore rely on alternative methods to explore regions unaccounted for in UKIRT.

High-resolution speckle images of Kepler-1649 were obtained on 2015 July 15 using the DSSI imaging camera mounted on the $8 \mathrm{~m}$ Gemini-N telescope. Observations with DSSI are taken simultaneously in two filters. This observation used a $692 \mathrm{~nm}$ center-wavelength filter with a $40 \mathrm{~nm}$ width and an $880 \mathrm{~nm}$ center-wavelength filter of width $50 \mathrm{~nm}$. The seeing was superb, near $0.4-0.5$ arcsec throughout with a total of 20 minutes spent collecting $60 \mathrm{~ms}$ frames on Kepler-1649. Details 


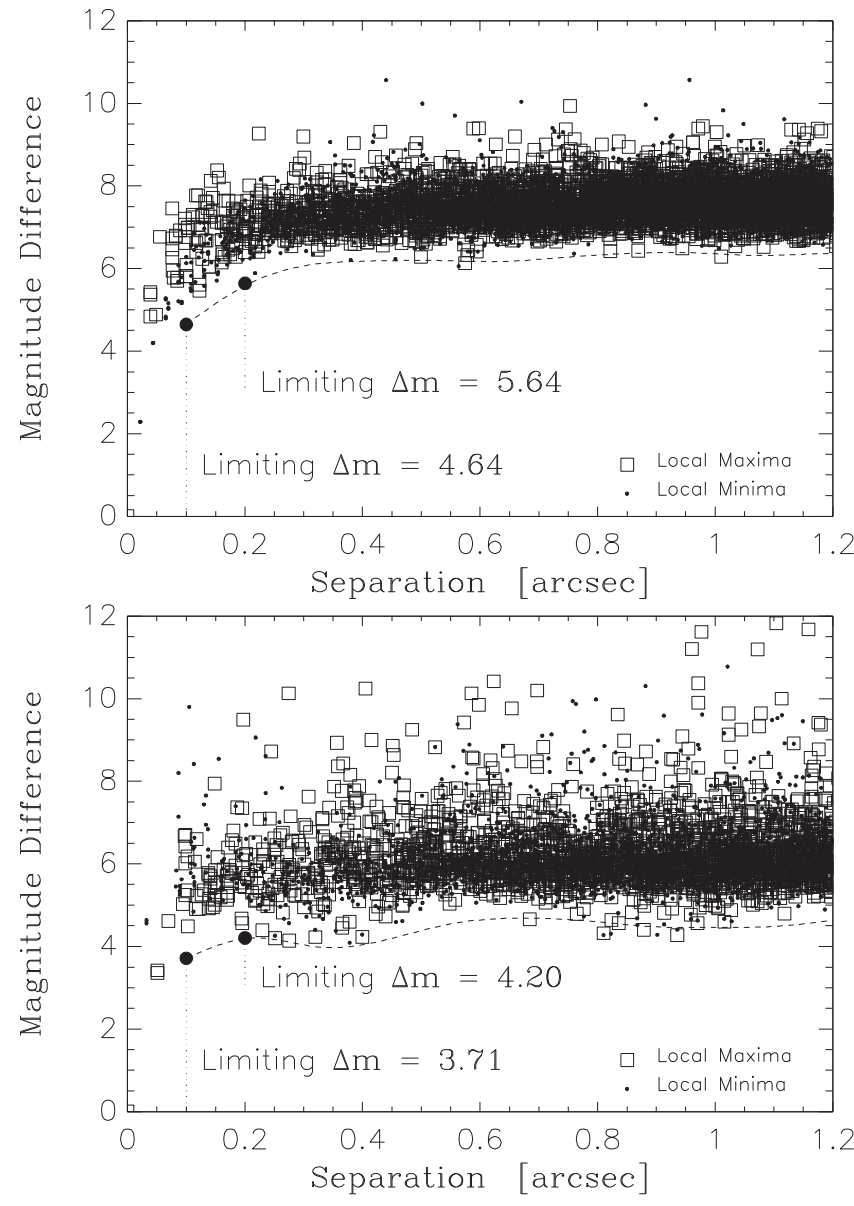

Figure 8. Detection limit analysis for the observation of Kepler-1649. In both plots, the dashed line represents the formal $5 \sigma$ limiting magnitude as a function of separation, as described in the text. The results in the 692 and $880 \mathrm{~nm}$ filters are shown in the top and bottom panels, respectively.

of the observational procedure and data reduction techniques are given in Horch et al. (2012).

Figure 8 shows the results from our two high-resolution speckle images, where the upper limit in magnitude as a function of separation is given by the dashed line. Kepler-1649 is a faint star for speckle observations, but both images nonetheless find at high significance that no companion star exists. The image at $692 \mathrm{~nm}$ reveals that at $5 \sigma$ no companion within a magnitude difference $\Delta K p \sim 5$ mag exists down to a spatial radius of $0.022 \mathrm{arcsec}$. The $880 \mathrm{~nm}$ image is somewhat poorer, finding that no companion exists at $5 \sigma$ to a $\Delta K p$ of near $4 \mathrm{mag}$ into $0.027 \mathrm{arcsec}$, as indicated by the green area of Figure 6.

Near-infrared adaptive optics (AO) images of Kepler-1649 were taken in the $J$-band $(1.248 \mu \mathrm{m})$ and $K$-band $(2.15 \mu \mathrm{m})$ filters. The images were taken using the NIRC2 imager on the Keck II Telescope on UT 2015 June 21 and are shown in Figure 9. Also shown are the sensitivity limits of the NIRC2 imager as a function of radial distance from the host star. As illustrated in the figures, no companions can be seen with $\mathrm{AO}$ imaging in any region within $\sim 00^{\prime \prime} 1-3^{\prime \prime}$ in the $J$ - and $K$-band filters to within a magnitude difference of 7.48 at 0.5 arcsec in the $J$ band. This data are also converted to Kepler magnitudes according to methods outlined in Howell et al. (2012). The cyan region of Figure 6 represents areas of parameter space that can be eliminated as companion hosting, according to our $\mathrm{AO}$ observations.

Given the data presented in Figure 6, a false-positiveinducing bound companion to Kepler-1649 can only exist in the parameter space indicated by the small white region, $\sim 5$ mag fainter than the target. Additionally, in order to remain consistent with our Kepler transit, a companion transit source must be a maximum of 5.8 mag fainter than our target star. From this information we assess the probability that the signal was induced by a planet transit around a star physically associated with Kepler-1649. We first determined the mass and radius of the faintest possible binary companion to Kepler-1649 using COND03 isochrone models (Baraffe et al. 2003). We computed the mass for a star 5.8 mag fainter than Kepler-1649 and found a lower limit of $0.05 M_{\odot}$ and a corresponding radius of $0.093 R_{\odot}$.

According to the isochrone models, a companion with a mass of 0.05-0.07 $M_{\odot}$ would be between 4.028 and 5.390 mag fainter than our host star, placing it near the M star-brown dwarf boundary. Fitting these star parameters to transit curves from Kepler data reveals that a planet transiting around a companion would have a radius of $\approx 2.8-4.7 R_{\oplus}$. Such a scenario would be consistent with our observations; however, the prospect of the system existing in the first place is highly unlikely given the low occurrence rates $\left(\leqslant 0.15 \mathrm{star}^{-1}\right)$ of large planets around cool stars (Berta et al. 2013).

Given the low likelihood of a nearby, bound false-positive source, in conjunction with our nondetection of a confounding background binary system, we verify that the transit signal around Kepler-1649 is due to a planet, Kepler-1649b, orbiting the system.

\section{Discussion}

We present the discovery and planetary confirmation of Kepler-1649b, an approximately Earth-sized planet in a 9-day orbit around a nearby M5V star receiving an incident flux of $2.30 \pm 0.65$ relative to Earth. We cannot derive a mass estimate for the planet from photometric data alone and currently do not have constraints from transit timing analysis or radial velocity measurement. Additionally, Kepler-1649b is too small to induce a detectable "wobble" in its host star, which could provide future constraints on its mass. We therefore make no conclusions about mass or composition in this paper. Planets with sizes comparable to Earth, however, have a high likelihood of being rocky (Rogers 2015).

Kepler-1649b is comparable in size and host star to Kepler186f, an Earth-sized exoplanet discovered to orbit in the habitable zone of an M dwarf (Quintana et al. 2014). Both planets orbit cool stars and thus exist in systems that are significantly different from that of the terrestrial planets in our solar system. Kepler-186f orbits a star that is about half a solar radius with a 130-day period, while Kepler-1649b orbits with an 8.7-day period around a star that is about one-quarter the size of our Sun. Because of this, the two planets may be more prone to effects of host star variability such as flares and coronal mass ejections than Earth and Venus. They also receive comparatively low energy radiation, due to a shift in the spectral energy distribution for $M$ dwarfs relative to the Sun. Furthermore, because Kepler-1649b and Kepler-186f have orbits much closer in than those of Venus and Earth, they may be subject to larger tidal effects from their host star. These effects may include tidal heating, synchronous rotation, and 

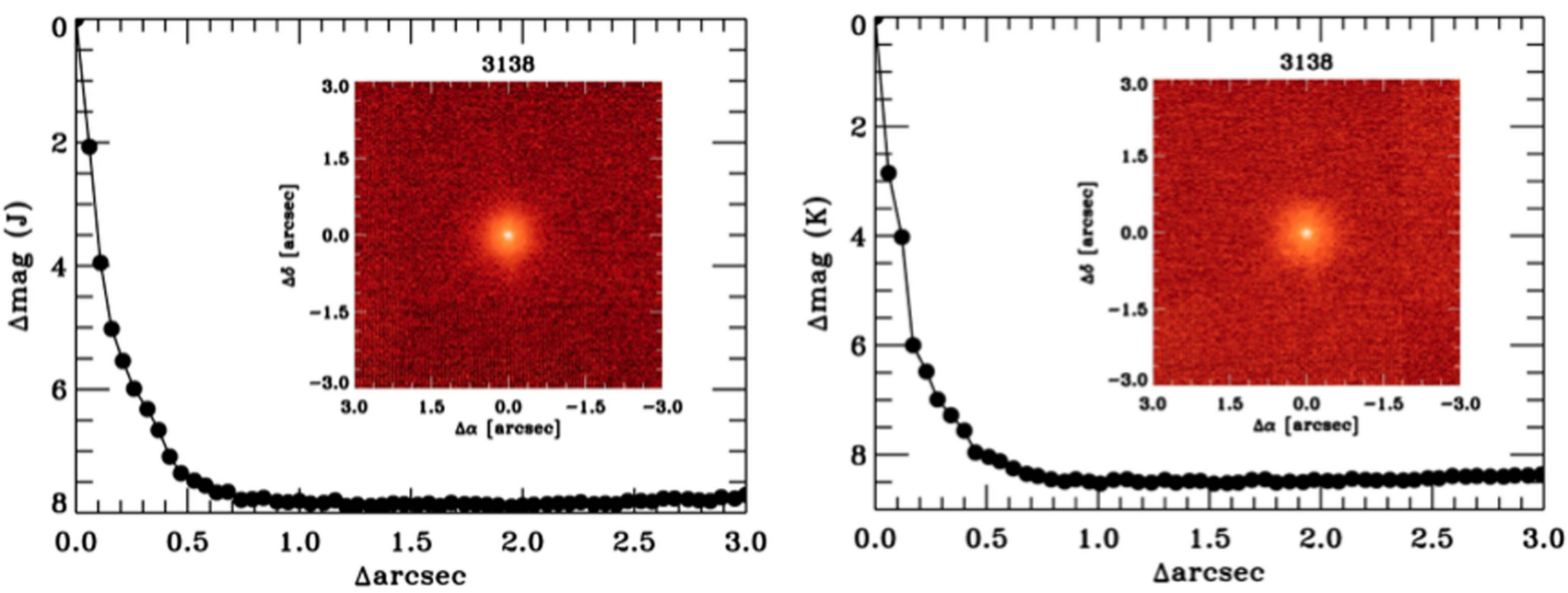

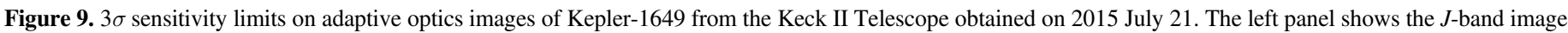
and sensitivity curve, and the right panel shows the same for the $K$-band filter. Neither image reveals a possible bound companion.

tidal locking, which can produce a significant effects on the planets' seasons and geologic activity.

Regardless, due to their size and incident flux, planets like Kepler-1649b and Kepler-186f are good candidates for Earthand Venus-analog studies. In terms of insolation, Kepler-1649b is too hot to reside within its star's habitable zone and instead is in the so-called "Venus Zone," a Venusian analog to the habitable zone as described in Kane et al. (2014). The discovery of Kepler-1649b thus highlights the relatively high abundance of terrestrial planets that may have runaway greenhouse surface environments, lending itself to future studies surrounding exoplanet atmospheres and habitability.

Distinguishing between Earth and Venus analogs is becoming especially important as the ongoing $K 2$ mission, like Kepler, discovers and studies more and more Earth-sized, nearhabitable zone planets (Demory et al. 2016). It will also remain important for this same reason as the upcoming Transiting Exoplanet Survey Satellite (TESS) mission preferentially detects and observes planets that are close to their host star (Ricker et al. 2014). These discoveries will lend themselves well to observations with the James Webb Space Telescope (JWST), which will have the potential to probe the atmospheres of planets such as Kepler-1649b and ultimately constrain their habitability (Greene et al. 2016). Such observations can help us understand the correlation between insolation flux (2.30 $\pm 0.65 S_{\oplus}$ for Kepler-1649b, easily observable for missions like Kepler) and habitability. We can also learn about other factors, like additional undetected planets in the system or tidal effects due to proximity to the host star, that may further contribute to a Venus-like climate (Barnes et al. 2013).

Similar to planets that lie within a star's habitable zone, confirmation of surface conditions of Kepler-1649b would require a detailed spectroscopic analysis of the atmosphere. Facilities capable of extracting such measurements for this planet are unlikely to be available in the near term. The detection of Venus-analog atmospheres via methods listed above presents a significant challenge due to the opacity of the Venusian atmosphere, though there are distinguishing features at high altitudes, including carbon dioxide absorption combined with an upper haze layer with sulfuric acid (Ehrenreich et al. 2012; Barstow et al. 2016). A cloud-dominated atmosphere also produces large scattering and reflection effects that translate into a relatively high geometric albedo, producing another source of evidence linking the atmosphere to a runaway greenhouse (Kane et al. 2013). Adopting a Venusian geometric albedo of 0.65 , we calculate a flux ratio amplitude between the planet and the host star of $5.2 \times 10^{-7}$. For comparison, Venus in our solar system produces a phase amplitude of $2.0 \times 10^{-9}$ (Kane \& Gelino 2013). The predicted phase variation amplitude of the planet is beneath the noise threshold of the Kepler photometry, but it could be examined as a diagnostic from follow-up observations of similar Venusanalog candidates.

Further constraints on the stellar parameters of Kepler-1649 are needed to increase the accuracy of our predicted planet properties. Fortunately, the GAIA spacecraft is a space-based telescope capable of measuring distances to nearby systems like Kepler-1649 by taking precise parallax measurements (Stassun et al. 2016). Such distance measurements will help place constraints on the luminosity of Kepler-1649 and thereby further increase the accuracy of the star and planet parameter calculations summarized in Table 1.

The discovery of Kepler-1649b is part of a larger movement toward confirmation and characterization of a variety of Earthsized exoplanets, with the ultimate goal of understanding what factors place constraints on habitability. Most of these planets have orbital periods measured to high precision, allowing us to calculate the flux received by the planet from its host star. As a result, determining the correlation between incident flux and atmospheric compositions would be highly useful in assessing the habitability of known exoplanets. More specifically, determining the compositions and atmospheres of planets like Kepler-1649b and Kepler-186f, two planets that together span a wide range of distances within the habitable zones of M dwarfs, will be useful in understanding the nature of habitable zone boundaries for such star types. Future missions like K2, TESS, and JWST, as described above, will make these studies possible and therefore lend themselves to a better understanding of conditions required for exoplanet habitability.

J.F.R. acknowledges NASA grants NNX12AD21G and NNX14AB82G issued through the Kepler Participating Scientist Program. The research presented in this paper includes data collected by the Kepler mission. This research 
has made use of the NASA Exoplanet Archive, which is operated by the California Institute of Technology, under contract with the National Aeronautics and Space Administration under the Exoplanet Exploration Program. Data from the Mikulski Archive for Space Telescopes (MAST) were used for this research as well. Data reduction was done using Image Reduction and Analysis Facility (IRAF) routines. E.V.Q. was supported by an appointment to a NASA Postdoctoral Program Senior Fellowship at NASA Ames Research Center, administered by Universities Space Research Association under contract with NASA. B.B. acknowledges financial support from the European Commission in the form of a Marie Curie International Outgoing Fellowship (PIOF-GA-2013-629435). Some of the data presented herein were obtained at the W. M. Keck Observatory, which is operated as a scientific partnership among the California Institute of Technology, the University of California, and the National Aeronautics and Space Administration. The Observatory was made possible by the generous financial support of the W. M. Keck Foundation. The authors wish to recognize and acknowledge the very significant cultural role and reverence that the summit of MaunaKea has always had within the indigenous Hawaiian community. We are most fortunate to have the opportunity to conduct observations from this mountain.

\section{References}

Allard, F., Homeier, D., \& Freytag, B. 2011, in ASP Conf. Ser. 448, 16th Cambridge Workshop on Cool Stars, Stellar Systems, and the Sun, ed. C. Johns-Krull, M. K. Browning, \& A. A. West (San Francisco, CA: ASP), 91

Baraffe, I., Chabrier, G., Barman, T. S., Allard, F., \& Hauschildt, P. H. 2003, A\&A, 402, 701

Barclay, T., Burke, C. J., Howell, S. B., et al. 2013a, ApJ, 768, 101

Barclay, T., Rowe, J. F., Lissauer, J. J., et al. 2013b, Natur, 494, 452

Barnes, R., Mullins, K., Goldblatt, C., et al. 2013, AsBio, 13, 225

Barstow, J. K., Aigrain, S., Irwin, P. G. J., Kendrew, S., \& Fletcher, L. N. 2016, MNRAS, arXiv: 1602.08277

Batalha, N. M., Borucki, W. J., Koch, D. G., et al. 2010, ApJL, 713, L109

Batalha, N. M., Rowe, J. F., Bryson, S. T., et al. 2013, ApJS, 204, 24

Berta, Z. K., Irwin, J., \& Charbonneau, D. 2013, ApJ, 775, 91

Borucki, W. J., Agol, E., Fressin, F., et al. 2013, Sci, 340, 587

Borucki, W. J., Koch, D., Basri, G., et al. 2010, Sci, 327, 977

Boyajian, T. S., von Braun, K., van Belle, G., et al. 2012, ApJ, 757, 112

Brown, T. M., Latham, D. W., Everett, M. E., \& Esquerdo, G. A. 2011, arXiv: 1102.0342

Bryson, S. T., Jenkins, J. M., Gilliland, R. L., et al. 2013, PASP, 125, 889
Bryson, S. T., Jenkins, J. M., Klaus, T. C., et al. 2010, Proc. SPIE, 7740, 77401D

Burke, C. J., Bryson, S. T., Mullally, F., et al. 2014, ApJS, 210, 19

Chaplin, W. J., Sanchis-Ojeda, R., Campante, T. L., et al. 2013, ApJ, 766, 101

Christiansen, J. L., Jenkins, J. M., Caldwell, D. A., et al. 2012, PASP, 124, 1279

Demory, B.-O., Queloz, D., Alibert, Y., Gillen, E., \& Gillon, M. 2016, ApJL, $825, \mathrm{~L} 25$

di Stefano, R. 2012, ApJS, 201, 20

Dotter, A., Chaboyer, B., Jevremovič, D., et al. 2008, ApJS, 178, 89

Ehrenreich, D., Vidal-Madjar, A., Widemann, T., et al. 2012, A\&A, 537, L2

Everett, M. E., Howell, S. B., \& Kinemuchi, K. 2012, PASP, 124, 316

Everett, M. E., Howell, S. B., Silva, D. R., \& Szkody, P. 2013, ApJ, 771, 107

Ford, E. B. 2005, AJ, 129, 1706

Gaidos, E., Mann, A. W., Kraus, A. L., \& Ireland, M. 2016, MNRAS, 457, 2877

Greene, T. P., Line, M. R., Montero, C., et al. 2016, ApJ, 817, 17

Gregory, P. C. 2011, MNRAS, 410, 94

Greiss, S., Steeghs, D., Gänsicke, B. T., et al. 2012, AJ, 144, 24

Horch, E. P., Howell, S. B., Everett, M. E., \& Ciardi, D. R. 2012, AJ, 144, 165

Horch, E. P., Howell, S. B., Everett, M. E., \& Ciardi, D. R. 2014, ApJ, 795, 60

Howell, S. B., Rowe, J. F., Bryson, S. T., et al. 2012, ApJ, 746, 123

Huber, D., Chaplin, W. J., Christensen-Dalsgaard, J., et al. 2013, ApJ, 767, 127

Huber, D., Silva Aguirre, V., Matthews, J. M., et al. 2014, ApJS, 211, 2

Jenkins, J. M., Caldwell, D. A., \& Borucki, W. J. 2002, ApJ, 564, 495

Kane, S. R., Barclay, T., \& Gelino, D. M. 2013, ApJL, 770, L20

Kane, S. R., \& Gelino, D. M. 2013, ApJ, 762, 129

Kane, S. R., Kopparapu, R. K., \& Domagal-Goldman, S. D. 2014, ApJL, 794, L5

Lépine, S., \& Shara, M. M. 2005, AJ, 129, 1483

Mandel, K., \& Agol, E. 2002, ApJL, 580, L171

Mann, A. W., Brewer, J. M., Gaidos, E., Lépine, S., \& Hilton, E. J. 2013a, AJ, 145,52

Mann, A. W., Feiden, G. A., Gaidos, E., Boyajian, T., \& von Braun, K. 2015, ApJ, 804, 64

Mann, A. W., Gaidos, E., \& Ansdell, M. 2013b, ApJ, 779, 188

Quintana, E. V., Barclay, T., Raymond, S. N., et al. 2014, Sci, 344, 277

Rappaport, S., Barclay, T., DeVore, J., et al. 2014, ApJ, 784, 40

Reid, I. N., Brewer, C., Brucato, R. J., et al. 1991, PASP, 103, 661

Ricker, G. R., Winn, J. N., Vanderspek, R., et al. 2014, Proc. SPIE, 9143 914320

Rogers, L. A. 2015, ApJ, 801, 41

Rowe, J. F., Bryson, S. T., Marcy, G. W., et al. 2014, ApJ, 784, 45

Rowe, J. F., Coughlin, J. L., Antoci, V., et al. 2015, arXiv:1501.07286

Smith, J. C., Stumpe, M. C., van Cleve, J. E., et al. 2012, PASP, 124, 1000

Stassun, K. G., Collins, K. A., \& Gaudi, B. S. 2016, arXiv:1609.04389

Still, M., \& Barclay, T. 2012, PyKE: Reduction and analysis of Kepler Simple Aperture Photometry data, Astrophysics Source Code Library, ascl: 1208.004

Stumpe, M. C., Smith, J. C., Catanzarite, J. H., et al. 2014, PASP, 126, 100

Stumpe, M. C., Smith, J. C., van Cleve, J. E., et al. 2012, PASP, 124, 985

Teske, J. K., Ghezzi, L., Cunha, K., et al. 2015, ApJL, 801, L10

Torres, G., Kipping, D. M., Fressin, F., et al. 2015, ApJ, 800, 99 